\title{
Atualizações em Infeções Sexualmente Transmissíveis
}

\author{
Alexandre Miroux Catarino', João Borges-Costa ${ }^{2,3,4}$ \\ 'Médico Interno de Dermatovenereologia/Resident of Dermatology and Venereology, Serviço de Dermatologia, Centro Hospitalar \\ de Lisboa Ocidental, Lisboa, Portugal \\ ${ }^{2}$ Assistente Hospitalar de Dermatovenereologia / Consultant, Dermatology and Venereology, Centro Hospitalar Lisboa Norte, \\ Lisboa, Portugal \\ ${ }^{3}$ Professor Auxiliar Convidado de Dermatologia e Venereologia / Associated Professor, Dermatology and Venereology, Faculdade de \\ Medicina da Universidade de Lisboa, Lisboa, Portugal \\ ${ }^{4}$ Professor Auxiliar Convidado de Microbiologia / Professor, Microbiology, Instituto de Higiene e Medicina Tropical da Universidade \\ Nova de Lisboa, Lisboa, Portugal
}

RESUMO - As infeções sexualmente transmissíveis são uma importante área de saúde pública, sendo necessário que os clínicos estejam alerta para o seu diagnóstico. Abordamos três temas que são atualmente pertinentes: o vírus Zika como nova infeção sexualmente transmissível, hepatites, incluindo a epidemia de hepatite $\mathrm{C}$ em homens que têm sexo com homens infetados com o vírus da imunodeficiência humana e o surto de hepatite $A$ de transmissão sexual que se tem verificado na Europa, e as resistências antimicrobianas da Neisseria gonorrhoeae.

PALAVRAS-CHAVE - Doenças Sexualmente Transmissíveis; Infecção pelo Zika Vírus; Infecções por VIH; Hepatite A; Hepatite C; Hepacivirus; Neisseria gonorrhoeae; Vírus Zika.

\section{Update on Sexually Transmitted Infections}

ABSTRACT - Sexually transmitted infections are an important area of public health, requiring clinicians to be alert to their diagnosis. We present three issues that we consider pertinent: the Zika virus as a new sexually transmitted infection, hepatitis, including the epidemic hepatitis C infection in men who have sex with men that are HIV positive and the sexually transmitted hepatitis A outbreak verified in Europe, and the antimicrobial resistance of Neisseria gonorrhoeae.

KEYWORDS - Hepacivirus; Hepatite A; Hepatitis A Virus; Hepatitis C; HIV Infections; Neisseria gonorrhoeae; Sexually Transmitted Diseases; Zika Virus; Zika Virus Infection.

\section{INTRODUÇÃO}

As infeções sexualmente transmissíveis (IST) são uma importante área de saúde pública, sendo necessário que os clínicos estejam alerta para o seu diagnóstico. A globalização com o grande número de pessoas que viajam de umas regiões para outras, quer em viagens de turismo, de trabalho ou migrações, associada a alteração de comportamentos sexuais e facilidade de contacto com possíveis parceiros através das novas tecnologias promove alterações rápidas da epidemiologia destas doenças. Na última década tem-se verificado um aumento das IST, sobretudo em grupos particulares de risco que exigem novas medidas preventivas.
Selecionámos três temas que achamos pertinentes na atualidade: o vírus Zika como nova IST, hepatites, incluindo a epidemia de hepatite $C$ em homens que têm sexo com homens $(\mathrm{HSH})$ infetados com o vírus da imunodeficiência humana $(\mathrm{VIH})$ e o surto de hepatite $\mathrm{A}$ de transmissão sexual que se tem verificado na Europa, e as resistências antimicrobianas da Neisseria gonorrhoeae.

\section{Zika - Nova IST}

O vírus Zika é um arbovírus RNA, pertencente ao género Flavivirus da família Flaviridae transmitido por mosquito. Foi pela primeira vez isolado em 1947 na floresta Zika no
Correspondência: Alexandre Miroux Catarino

E-mail: alexandre_catarino@hotmail.com
Recebido/Received 07 Março/March 2017

Aceite/Accepted

02 Abril/April 2017 
Tabela 1 - Recomendações da OMS para prevenção da transmissão sexual do vírus Zika ${ }^{13}$

\begin{tabular}{c|l}
\hline \multirow{2}{*}{$\begin{array}{c}\text { Regiões com transmissão } \\
\text { ativa do vírus por mosquito. }\end{array}$} & Uso do preservativo durante toda a gravidez. \\
\cline { 2 - 2 } & Acesso a contraceção de emergência se relação sexual desprotegida e possibilidade de infeção por Zika. \\
\hline \multirow{2}{*}{$\begin{array}{c}\text { Regiões sem transmissão } \\
\text { ativa do vírus. }\end{array}$} & $\begin{array}{l}\text { Homem ou mulher devem adotar medidas de proteção durante } 6 \text { meses para evitar transmissão sexual do } \\
\text { vírus, após regresso de região onde ocorre transmissão por mosquito. }\end{array}$ \\
\cline { 2 - 2 } $\begin{array}{c}\text { Se for planeada uma gravidez devem esperar-se } 6 \text { meses até tentativa de conceção, após regresso de região } \\
\text { onde ocorre transmissão por mosquito. }\end{array}$ & $\begin{array}{l}\text { Parceiro sexual de grávida que venha de região com transmissão ativa deve adotar abstinência ou medidas de } \\
\text { proteção durante o restante período de gestação. }\end{array}$ \\
\cline { 2 - 2 }
\end{tabular}

Uganda, num macaco rhesus. ${ }^{1} \mathrm{O}$ primeiro caso de infeção pelo vírus Zika foi reportado na Nigéria em 1954, e até 2007 apenas se reportaram 14 casos ocorridos entre África e Sudeste Asiático. ${ }^{2}$ Em 2015, a transmissão de Zika no Brasil foi confirmada e provocou uma epidemia estimada em 440000 - 1300000 casos. O vírus tem-se disseminado de forma rápida e mais de 73 países registaram casos de infeção por mosquito e 12 relataram casos de transmissão entre humanos. ${ }^{2,3}$ Atualmente, as principais regiões com casos autóctones são a América Central, a América do Sul e o Sudeste Asiático, sendo que em Angola tem-se verificado transmissão esporádica do vírus. ${ }^{4}$

A infeção por Zika tem um período de incubação de 3 a 12 dias e em mais de $80 \%$ é assintomática. A infeção sintomática tipicamente é ligeira e tem uma duração média de 2 a 7 dias, caracterizando-se por febre baixa, associada a cefaleia, artralgias, mialgias, acompanhada de um exantema maculopapular que habitualmente surge 3 a 4 dias após a febre. $O$ exantema localiza-se inicialmente na face/ região cervical e posteriormente dissemina-se pelo tronco e membros, persiste entre 2 a 12 dias e tem uma duração habitual de 6 dias. Pode envolver as palmas e plantas e ser pruriginoso. ${ }^{5,6}$ A síndrome de Guillain-Barré tem sido uma complicação major associada à infeção pelo vírus Zika. ${ }^{7,8}$ Outra complicação major da infeção pelo vírus ocorre nas mulheres grávidas. Apesar de a doença não ser mais grave nesta população, está demonstrada a relação causal entre infeção pré-natal e microcefalia e/ou outras anormalidades do desenvolvimento neurológico, na designada síndrome congénita do Zika. ${ }^{8}$ Esta será a primeira vez que um vírus transmitido por mosquito está associado a malformações congénitas.

As várias formas de transmissão do vírus ajudam a explicar sua rápida e extensa distribuição geográfica. A principal forma de transmissão ocorre através da picada de diferentes tipos de mosquito Aedes, sendo os mais importantes o Aedes aegypti e o Aedes albopictus. Outras formas de transmissão conhecidas são a transmissão sexual, transmissão vertical (transplacentar e durante o parto) e transfusional. Suspeita-se ainda da possível transmissão por outros fluídos orgânicos (suor, saliva)..$^{2,9}$
A importância da transmissão sexual ainda não está completamente elucidada, mas tem sido objeto de estudo pelo risco que representa a infeção de uma mulher grávida e por ser uma forma de transmissão possível em locais onde não está presente o mosquito Aedes, através de pessoas infetadas pelo vírus nos locais endémicos. A transmissão mais comummente descrita tem sido de homem sintomático para mulher. Foi descrita transmissão sexual do vírus até 41 dias após os sintomas iniciais, detetando-se presença no sémen de partículas virais infetantes até 69 dias e RNA viral até 188 dias pós infeção. $O$ trato urogenital masculino poderá funcionar como um reservatório do vírus. ${ }^{29-11}$

As últimas recomendações da Organização Mundial de Saúde (OMS) sobre o risco de transmissão sexual datam de setembro de 2016 (Tabela 1). O Centers for Disease Control and Prevention (CDC) difere no facto de considerar que a mulher deve aguardar apenas 8 semanas antes da tentativa de conceção se esteve numa região com transmissão ativa do vírus. No homem o CDC atualizou a recomendação para os 6 meses de espera antes de tentativa de conceção devido aos novos dados que apontam para permanência do vírus durante mais tempo no sémen. ${ }^{12}$ As diretivas da Direção Geral de Saúde (Comunicado n C106_04_v1 de $22 / 12 / 2016)$ vão de encontro às orientações da $C D C$, recomendando que após o regresso de países onde ocorre transmissão do vírus por mosquito, o homem deva adotar medidas de proteção durante 6 meses e a mulher durante 8 semanas. Estas recomendações têm evoluído à medida que são conhecidos novos dados sobre a transmissão sexual do vírus.

\section{Hepatites}

a. Hepatite $\mathrm{C}-\mathrm{A}$ epidemia em HSH infetados com $\mathrm{VIH}$

$O$ vírus da hepatite $\mathrm{C}(\mathrm{VHC})$ é um agente transmitido pelo sangue primariamente por exposição parentérica, nomeadamente por partilha de agulhas por utilizadores de drogas injetáveis, transfusão de produtos contaminados e acidentes com dispositivos médicos contaminados. ${ }^{14} \mathrm{~A}$ transmissão sexual do vírus é considerada uma forma pouco eficaz de transmissão do vírus, ${ }^{15}$ principalmente em heterossexuais. No entanto, na última década, tem-se verificado 
Tabela 2 - Recomendação de Rastreio para infeção VHC aguda segundo a European AIDS Treatment Network ${ }^{30}$

- Todos os indivíduos diagnosticados de novo com VIH

- $\mathrm{HSH}$ e $\mathrm{VIH}+$ em risco de contrair hepatite $\mathrm{C}$ aguda devem ser rastreados com ALT semestralmente e com anticorpos anti-VHC anualmente

- VIH + com nova IST diagnosticada ou uso contínuo de drogas injetáveis devem ser rastreados 3 meses após diagnóstico/última exposição

- Um teste de ácidos nucleicos para pesquisa de RNA de VHC deve ser realizado se suspeita de infeção VHC aguda

um aumento de incidência de $\mathrm{VHC}$ nos $\mathrm{HSH}$ infetados com $\mathrm{VIH}$, com transmissão através das mucosas. ${ }^{16}$

Em 2004-2005 foi reportado um aumento significativo na incidência de $\mathrm{VHC}$ em HSH e VIH + na Europa Ocidental. ${ }^{17,18}$ Nos Países Baixos, um estudo de seguimento de HSH que recorreram a uma clínica especializada em IST revelou um aumento de prevalência de $15 \%$ em 2007 para $21 \%$ em 2008 no grupo dos VIH +, em comparação a prevalências estimadas de $1-4 \%$ antes de 2000. Dos novos casos de infeção, somente $5 \%$ dos indivíduos referia uso de drogas injetáveis. Por oposição, a prevalência da infeção no grupo dos $\mathrm{HSH}$ e VIH - era de apenas $0,4 \% .{ }^{19}$ No Reino Unido a prevalência subiu de 4,1 em 2002-2003, para $7,7 \%$ em 2008 neste mesmo grupo dos $\mathrm{HSH}$ e $\mathrm{VIH}+.20,21$

Outra alteração recente é o número elevado de reinfeções nesta população. Um estudo em Amsterdão mostrou que 11 de $56 \mathrm{HSH}$ e $\mathrm{VIH}$ + tratados para o $\mathrm{VHC}$, com resposta viral sustentada pós tratamento, foram reinfectados. ${ }^{22}$

Há fatores biológicos e outros relacionados com comportamentos que parecem estar associados a esta forma de transmissão sexual do $\mathrm{VHC} .{ }^{23}$ Nos fatores biológicos salientam-se a infeção VIH e a co-existência de outras IST. A infeção VIH aumenta a infeciosidade e a suscetibilidade ao $\mathrm{VHC}$, estando associada a aumento da carga viral de VHC, resposta humoral e celular atenuada ao vírus e maior probabilidade de eliminar vírus no sémen. ${ }^{24} \mathrm{~A}$ co-existência de outras IST, ao provocarem disrupção da integridade da mucosa facilitam a transmissão do vírus. Dentro dos fatores comportamentais destacam-se:

1. Comportamento seroadaptativo, em que há procura de relações sexuais sem preservativo com parceiros $\mathrm{VIH}$ concordantes, sem preocupação face a outras IST;

2. Práticas sexuais traumáticas para a mucosa, principalmente da mucosa retal com hemorragia associada ${ }_{i}^{25}$

3. Uso de drogas recreacionais não injetáveis administradas via mucosa, quer por fomentarem a adoção de comportamentos sexuais de risco quer por eventual efeito facilitador na transmissão do VHC, nomeadamente através da hiperémia que provocam e pela partilha do objeto utilizado na administração.
Será importante educar esta população para a evicção de práticas sexuais traumáticas ou partilhas de objeto com sangue. $O$ uso do preservativo ou luvas não evita a transmissão do vírus se estiverem contaminados com sangue e forem usados em diferentes parceiros no contexto de sexo em grupo. ${ }^{25}$

b. Hepatite A - Surto de transmissão sexual na Europa

Surtos de hepatite A por transmissão sexual têm também sido descritos no grupo dos $\mathrm{HSH} .{ }^{26}$ Desde fevereiro de 2016 foram notificados 287 casos confirmados de hepatite A distribuídos por 13 países europeus, incluindo Portugal, a maioria ocorrendo em HSH.27-29 É importante promover a vacinação contra a hepatite $A$ neste grupo populacional.

\section{Resistências antimicrobianas da Neisseria go-} norrhoeae

A Neisseria gonorrhoeae (NG) é um diplococo gram-negativo, aeróbio, intra-celular, que infeta apenas o ser humano. É o agente etiológico da gonorreia e é transmitido maioritariamente por via sexual através do contacto direto de membranas mucosas. A infeção por NG provoca uretrite no homem e cervicite na mulher, sendo que pode ser assintomática em até $10 \%$ nos homens e $50 \%$ nas mulheres. A infeção da orofaringe ou rectal habitualmente é assintomática. ${ }^{31}$ As estimativas da OMS em 2012 apontavam para uma incidência mundial de 78 milhões de casos de gonorreia, sendo a segunda IST bacteriana mais frequente na Europa a seguir à Chlamydia trachomatis. ${ }^{32}$

A NG é uma preocupação de saúde pública global pela possibilidade de vir a transformar-se numa bactéria resistente a todos os fármacos disponíveis. Esta tem vindo a desenvolver resistência a todos os antibióticos introduzidos para o seu tratamento desde 1930, através da capacidade de alterar a sua expressão génica de acordo com as pressões do meio, troca de informação genética com outros microorganismos da flora humana e de mecanismos fisiológicos de resistência aos antibióticos. ${ }^{33,34}$

Nas guidelines europeias de 2009 as cefalosporinas de largo espectro (ESC) em monoterapia (ceftriaxone intramuscular e cefixime oral) eram o tratamento de primeira linha. ${ }^{35}$ No entanto, o registo de casos de falência terapêutica e a emergência de estirpes de NG resistentes levou a que fossem adotados planos de contingência pelas autoridades de saúde internacionais. Segundo a OMS, a terapêutica de $1^{a}$ linha deve ser eficaz em mais de 95\%, e em vários países europeus mais de $5 \%$ dos isolamentos de NG eram resistentes ao cefixime nas doses recomendadas. ${ }^{36} \mathrm{Em}$ resposta, foi adotada a terapêutica dupla de ceftriaxone com azitromicina ${ }^{37}$ e a redução de resistência ao cefixime de 2010 a 2014 parece corroborar a eficácia desta estratégia. ${ }^{36}$ No entanto, as doses de ceftriaxone e azitromicina têm sido sucessivamente aumentadas nas revisões das guidelines. A resistência à azitromicina tem vindo a aumentar e em 2014, 7,9\% dos isolamentos demonstravam NG resistente (concentração inibitória mínima $>0,5 \mathrm{mg} / \mathrm{L}$ ). ${ }^{36}$ 
Tabela 3 - Guidelines Europeias de 2012 para o tratamento de infeções anogenitais não complicadas a NG ${ }^{37}$

\begin{tabular}{|c|c|}
\hline Tratamento recomendado ( $1^{a}$ linha) & Ceftriaxone (500 mg x $1 \mathrm{IM})+$ Azitromicina (2 gr x $1 \mathrm{PO})$ \\
\hline \multirow{2}{*}{ Alternativas } & Cefixime (400 mg x 1 PO) + Azitromicina (2 gr x 1 PO) ${ }^{1}$ \\
\hline & Espectinomicina $(2 \mathrm{~g} \times 1 \mathrm{IM})+$ Azitromicina $(2 \mathrm{gr} \times 1 \mathrm{PO})^{3}$ \\
\hline $\begin{array}{l}\text { Terapêutica recomendada se resistência a } \\
\text { ESC ou falência do tratamento de } 1^{a} \text { linha }\end{array}$ & Ceftriaxone (1 gr x 1 IM) + Azitromicina (2 gr x 1 PO) \\
\hline
\end{tabular}

Mais preocupantes são os casos descritos de resistência à terapêutica dupla, com particular preocupação para a dificuldade de erradicação da NG da orofaringe. ${ }^{38}$

Novos fármacos e associações têm vindo a ser experimentados in vivo e in vitro, incluindo ertapenem, solitromicina, avarofloxacina, tigeciclina, eravaciclina, dalvabancin, entre outros. Duas novas associações foram recentemente estudadas como alternativas terapêuticas caso as resistências ao ceftriaxone se tornarem generalizadas. A associação de gentamicina $240 \mathrm{mg}$ intramuscular mais azitromicina 2 gr per os e a associação de gemifloxacina $320 \mathrm{mg}$ per os mais azitromicina $2 \mathrm{gr}$ per os obtiveram taxas de cura de 100 e 99,5\% respetivamente. ${ }^{39}$ A espectinomicina, utilizada como primeira linha na década de 1960, é eficaz para o tratamento de NG anogenital, mas não para a NG presente na orofaringe e é atualmente uma alternativa terapêutica. ${ }^{40}$ Em Portugal não está disponível desde 2013.

As guidelines do CDC de 2015 recomendam a associação de ceftriaxone $250 \mathrm{mg}$ mais azitromicina $1 \mathrm{gr}$ para o tratamento de infeções anogenitais não complicadas a NG. ${ }^{41}$ As guidelines europeias recomendam doses superiores dos mesmos fármacos de acordo com os perfis e evolução das resistências na Europa, pelo que consideramos serem aquelas que devem ser seguidas. ${ }^{37}$

O cumprimento das guidelines (Tabela 3 ) associada a programas de vigilância adequados deve ser a regra de forma a evitar o desenvolvimento de estirpes multirresistentes. ${ }^{37}$

\section{CONCLUSÃO}

As IST são uma área importante da atuação da médica, exigindo um constante atualizar de conhecimentos por parte dos clínicos que lidam com este tipo de patologia quer em consultas de venereologia, serviço de urgência ou consulta do viajante, entre outras. Pelas suas caraterísticas, as alterações epidemiológicas são rápidas e exigem que as autoridades tenham programas de vigilância eficazes de forma a responder em tempo útil aos novos desafios.

Em relação à infeção pelo vírus Zika, os indivíduos que viajam ou regressam de áreas endémicas onde ocorre transmissão por mosquito devem estar alerta para esta nova realidade, os riscos que implica a infeção pré-natal quando afeta mulheres grávidas e a possibilidade de transmissão sexual do vírus. A prevalência de IST é particularmente elevada no grupo de risco dos HSH, sendo importante compreensão desta realidade e a adoção de políticas de saúde mais eficazes. A capacidade da NG em desenvolver resistência aos antimicrobianos tem sido uma constante, e tal como outras IST bacterianas, exige planos de vigilância que permitam ir adequando a terapêutica recomendada e que incentive os clínicos a seguir as guidelines para evitar a falência terapêutica e seleção de estirpes resistentes.

Conflitos de interesse: Os autores declaram não possuir conflitos de interesse.

Suporte financeiro: $O$ presente trabalho não foi suportado por nenhum subsídio ou bolsa.

Conflicts of interest: The authors have no conflicts of interest to declare.

Financing Support: This work has not received any contribution, grant or scholarship.

\section{REFERÊNCIAS}

1. Hayes EB. Zika virus outside Africa. Emerg Infect Dis. 2009; 15:1347-50.

2. Baud D, Musso D, Vouga M, Alves MP, Vulliemoz N. Zika virus: $A$ new threat to human reproduction. American Journal of Reproductive Immunology. 2017;77:2.

3. Bogoch, II, Brady OJ, Kraemer MU, German M, Creatore $M I$, Kulkarni MA, et al. Anticipating the international spread of Zika virus from Brazil. Lancet. 2016;387:3356.

4. European Centre for Disease Prevention and Control (ECDC). [accessed September 2016] http://ecdc.europa. eu/en/healthtopics/zika_virus_infection/zika-outbreak/ Pages/epidemiological-situation.aspx.

5. He A, Brasil P, Siqueira AM, Calvet GA, Kwatra SG. The emerging Zika virus threat: a guide for dermatologists. Am J Clin Dermatol. 2017;18:231-6.

6. Hajra A, Bandyopadhyay D, Hajra S. Zika virus: A global threat to humanity: A comprehensive review and current developments. North Am J Med Sci. 2016;8:123-8.

7. Watrin L, Ghawche F, Larre P, Neau JP, Mathis S, Fournier E. Guillain-Barre syndrome (42 cases) occurring during a Zika virus outbreak in French Polynesia. Medicine. 2016;95:e3257.

8. Kraver F, Riesen M, Reveiz L, Oladapo OT, Martinez-Vega $R$, Porgo TV, et al. Zika virus infection as a cause of 
congenital brain abnormalities and Guillain-Barre syndrome: systematic review. PLoS Med. 2017;14:e1002203.

9. Moreira J, Peixoto TM, Machado de Siqueira A, Lamas CC. Sexually acquired Zika virus: a systematic review. Clin Microbiol Infect. 2017 (in press).

10. Rossi SL, Tesh RB, Azar SR, Muruato AE, Hanley KA, Auguste AJ, et al. Characterization of a novel murine model to study Zika virus. Am J Trop Med Hyg. 2016;94:13629.

11. D'Ortenzio E, Matheron S, Yazdanpanah Y, de Lamballerie X, Hubert B, Piorkowski G, et al. Evidence of sexual transmission of Zika virus. N Engl J Med. 2016;374:21958.

12. Petersen EE, Meaney-Delman D, Neblett-Fanfair R, Havers F, Oduyebo T, Hills SL, et al. Update: interim guidance for preconception counseling and prevention of sexual transmission of Zika virus for persons with possible zika virus exposure - United States, September 2016. MMWR Morb Mortal Wkly Rep. 2016;65:1077-81.

13. World Health Organization. Prevention of sexual transmission of Zika vírus. [accessed September 2016] Available from: http://www.who.int/csr/resources/publications/zika/sexual-transmission-prevention/en/.

14. Chan DP, Sun HY, Wong HT, Lee SS, Hung CC. Sexually acquired hepatitis $C$ virus infection: a review. Int J Infect Dis. 2016;49:47-58.

15. Tohme RA, Holmberg SD. Is sexual contact a major mode of hepatitis $C$ virus transmission? Hepatology. 2010;52:1497-505.

16. Bradshaw D, Matthews G, Danta M. Sexually transmitted hepatitis $C$ infection: the new epidemic in MSM? Curr Opin Infect Dis. 2013;26:66-72.

17. Gambotti L, Batisse D, Colin-de-Verdiere N, Delaroque-Astagneau E, Desenclos JC, Dominguez S, et al. Acute hepatitis $\mathrm{C}$ infection in HIV positive men who have sex with men in Paris, France, 2001-2004. Euro Surveill. 2005; 10: $115-7$.

18. Gotz HM, van Doornum G, Niesters HG, den Hollander JG, Thio HB, de Zwart O. A cluster of acute hepatitis $C$ virus infection among men who have sex with men--results from contact tracing and public health implications. AIDS. 2005; 19:969-74.

19. Urbanus AT, van de Laar TJ, Stolte IG, Schinkel J, Heijman T, Coutinho RA, et al. Hepatitis $C$ virus infections among HIV-infected men who have sex with men: an expanding epidemic. AIDS. 2009;23:F1-7.

20. Dougan S, Balogun MA, Elford J, Brant LJ, Sinka K, Evans $B G$, et al. Can current national surveillance systems in England and Wales monitor sexual transmission of hepatitis $\mathrm{C}$ among HIV-infected men who have sex with men? BMC Public Health. 2007;7:7.

21. Price H, Gilson R, Mercey D, Copas A, Parry J, Nardone $A$, et al. Hepatitis $C$ in men who have sex with men in London a community survey. HIV Med. 2013;14:578-80.

22. Lambers FA, Prins M, Thomas X, Molenkamp R, Kwa D, Brinkman $K$, et al. Alarming incidence of hepatitis $C$ virus re-infection after treatment of sexually acquired acute hepatitis $C$ virus infection in HIV-infected MSM. AIDS. 2011 ;25:F21-7.

23. Danta M, Rodger AJ. Transmission of HCV in HIV-positive populations. Curr Opin HIV AIDS. $2011 ; 6: 451$-8.

24. Briat A, Dulioust E, Galimand J, Fontaine H, Chaix ML, Letur-Konirsch $\mathrm{H}$, et al. Hepatitis $\mathrm{C}$ virus in the semen of men coinfected with HIV-1: prevalence and origin. AIDS. 2005; 19:1827-35.

25. Schmidt AJ, Rockstroh JK, Vogel M, An der Heiden M, Baillot A, Krznaric I, et al. Trouble with bleeding: risk factors for acute hepatitis C among HIV-positive gay men from Germany a case-control study. PLoS One. $2011 ; 6: e 17781$.

26. Urbanus AT, van Houdt R, van de Laar TJ, Coutinho RA. Viral hepatitis among men who have sex with men, epidemiology and public health consequences. Euro SurveiII. $2009 ; 14 ; 47$.

27. Beebeejaun K, Degala S, Balogun K, Simms I, Woodhall SC, Heinsbroek E, et al. Outbreak of hepatitis A associated with men who have sex with men (MSM), England, July 2016 to January 2017. Euro Surveill. 2017;22:5.

28. Freidl GS, Sonder GJ, Bovee LP, Friesema IH, van Rijckevorsel GG, Ruijs WL, et al. Hepatitis A outbreak among men who have sex with men (MSM) predominantly linked with the EuroPride, the Netherlands, July 2016 to February 2017. Euro Surveill. 2017;22;8.

29. European Centre for Disease Prevention and Control (ECDC). Hepatitis A outbreaks in the EU/EEA mostly affecting men who have sex with men. [September 2016] Available from: http://ecdc.europa.eu/en/publications/ Publications/16-02-2017-RRA\%20UPDATE\%201-Hepatitis\%20A-United\%20Kingdom.pdf.

30. Acute hepatitis $\mathrm{C}$ in HIV-infected individuals: recommendations from the European AIDS Treatment Network (NEAT) consensus conference. AIDS. 2011 ;25:399-409.

31. Unemo M, Del Rio C, Shafer WM. Antimicrobial resistance expressed by neisseria gonorrhoeae: a major global public health problem in the $21^{\text {st }}$ century. Microbiol Spectr. 2016;4:3.

32. Newman L, Rowley J, Vander Hoorn S, Wijesooriya NS, Unemo M, Low N, et al. Global Estimates of the prevalence and incidence of four curable sexually transmitted infections in 2012 based on systematic review and global reporting. PLoS One. 2015;10:e0143304.

33. Unemo M, Shafer WM. Antimicrobial resistance in Neisseria gonorrhoeae in the 21 st century: past, evolution, and future. Clin Microbiol Rev. 2014;27:587-613.

34. Suay-Garcia B, Perez-Gracia MT. Drug-resistant Neisseria gonorrhoeae: latest developments. Eur J Clin Microbiol Infect Dis. 2017 (in press).

35. Bignell C. 2009 European (IUSTI/WHO) guideline on the diagnosis and treatment of gonorrhoea in adults. Int $\mathrm{J}$ STD AIDS. 2009;20:453-7.

36. European Centre for Disease Prevention and Control (ECDC). Gonococcal antimicrobial susceptibility 
surveillance in Europe ECDC SURVEILLANCE REPORT; 2014 [accessed September 2016] Available from: http:// ecdc.europa.eu/en/publications/Publications/gonococcal-antimicrobial-susceptibility-surveillance-Europe-2014.pdf.

37. Bignell C, Unemo M. 2012 European guideline on the diagnosis and treatment of gonorrhoea in adults. Int $\mathrm{J}$ STD AIDS. 2013;24:85-92.

38. Fifer $\mathrm{H}$, Natarajan $U$, Jones L, Alexander S, Hughes G, Golparian D, et al. Failure of dual antimicrobial therapy in treatment of gonorrhea. $\mathrm{N}$ Engl J Med. 2016;374:2504-6.
39. Kirkcaldy RD, Weinstock HS, Moore PC, Philip SS, Wiesenfeld HC, Papp JR, et al. The efficacy and safety of gentamicin plus azithromycin and gemifloxacin plus azithromycin as treatment of uncomplicated gonorrhea. Clin Infect Dis. 2014;59:1083-91.

40. Unemo M. Current and future antimicrobial treatment of gonorrhoea - the rapidly evolving Neisseria gonorrhoeae continues to challenge. BMC Infect Dis. 2015; 15:364.

41. Workowski KA, Bolan GA. Sexually transmitted diseases treatment guidelines, 2015. MMWR Recomm Rep. 2015;64:1-137. 